\title{
Thermal entanglement in the anisotropic Heisenberg XXZ model with the Dzyaloshinskii-Moriya interaction
}

\author{
Da-Chuang $\mathrm{Li}^{1 *}$, Xian-Ping Wang ${ }^{1}$, Zhuo-Liang $\mathrm{Cao}^{1,2 \dagger}$ \\ ${ }^{1}$ Key Laboratory of Opto-electronic Information Acquisition and Manipulation (Ministry of Education), \\ School of Physics \& Material Science, Anhui University, Hefei 230039 P. R. China \\ ${ }^{2}$ Department of Physics, Hefei Teachers College, Hefei 230061 P. R. China
}

\begin{abstract}
The entanglement is investigated in a two-qubit Heisenberg XXZ system with Dzyaloshinskii-Moriya(DM) interaction. It is shown that the entanglement can be efficiently controlled by the DM interaction parameter and coupling coefficient $J_{z}$. $D_{x}$ (the x-component parameter of the DM interaction) has a more remarkable influence on the entanglement and the critical temperature than $D_{z}$ (the z-component parameter of the DM interaction). Thus, by changing the direction of DM interaction, we can get a more efficient control parameter to increase the entanglement and the critical temperature.
\end{abstract}

PACS numbers: 03.67.Lx, 03.65.Ud, 75.10.Jm

\section{INTRODUCTION}

Entanglement has been extensively studied in recent years because it has the fascinating nonclassical nature of quantum mechanics, and it plays a key role in quantum information processing [1, 2]. The quantum entanglement in solid state systems such as spin chains is an important emerging field [3, 4, 5, 6], spin chains are natural candidates for the realization of entanglement, and spin has been researched in many other systems, such as superconductor [7, 8], quantum dots [9, 10, 11], and trapped ion [12, 13].

In order to characterize qualitatively and quantitatively the entanglement properties of condensed matter systems and apply them in quantum information, the thermal entanglement qualities in Heisenberg model have been extensively studied [14, 15, 16, 17, 18], and many schemes of teleportation via thermal entangled state have been reported [19, 20, 21, 22, 23]. In condensed matter systems, the Heisenberg chains have also been used to construct a quantum computer [24], perform quantum computation [25, 26, 27], and so on.

In those studies the spin-spin interaction was considered, but the spin-orbit coupling was rarely considered. Especially the influences of $\mathrm{x}$-component DM interaction parameter on the entanglement and the critical temperature have never been reported. In this paper we investigate the influence of Dzyaloshinskii-Moriya interaction parameter (arising from the spin-orbit coupling) and coupling coefficient $J_{z}$ on the entanglement of a two-qubit anisotropic Heisenberg XXZ spin chain. We show that the DM interaction parameter and the coupling coefficient $J_{z}$ are both efficient control parameters of entanglement, increasing them can enhance the entanglement or slow down the decreace of the entanglement. In addition, by analyzing we know that different component parameters of DM interaction have different influences on the entanglement and the critical temperature $T_{c}$, the parameter $D_{x}$ (x-component parameter of the DM interaction) has a more

\footnotetext{
*E-mail: dachuang@ahu.edu.cn

${ }^{\dagger}$ E-mail:zhuoliangcao@gmail.com (Corresponding Author)
}

remarkable influences than the parameter $D_{z}$ (z-component parameter of the DM interaction). So more efficient control parameter can be obtained by changing the DM interaction direction.

Our paper is organized as follows. In Section II, we introduce the Hamiltonian of the two-qubit anisotropic Heisenberg XXZ chain with the z-component parameter of the DM interaction, we calculate the concurrence of this system, analyze the influence of parameters on the entanglement in ground state and thermal state. In Section III, we similarly analyze the model of the two-qubit Heisenberg XXZ chain with the $\mathrm{X}$-component parameter of the DM interaction. Then we compare the influences of the two component parameters of the DM interaction on the entanglement in Section IV. Finally, in Section $\mathrm{V}$ a discussion concludes the paper.

\section{XXZ HEISENBERG MODEL WITH DM INTERACTION PARAMETER $D_{z}$}

The Hamiltonian $H$ for a two-qubit anisotropic Heisenberg $\mathrm{XXZ}$ chain with DM interaction parameter $D_{z}$ is

$$
H=J \sigma_{1}^{x} \sigma_{2}^{x}+J \sigma_{1}^{y} \sigma_{2}^{y}+J_{z} \sigma_{1}^{z} \sigma_{2}^{z}+D_{z}\left(\sigma_{1}^{x} \sigma_{2}^{y}-\sigma_{1}^{y} \sigma_{2}^{x}\right),
$$

where $J$ and $J_{z}$ are the real coupling coefficients, $D_{z}$ is the zcomponent parameter of DM interaction, $\sigma^{i}(i=x, y, z)$ are Pauli matrices. The coupling constants $J>0$ and $J_{z}>0$ correspond to the antiferromagnetic case, $J<0$ and $J_{z}<0$ correspond to the ferromagnetic case. This model is reduced to isotropic XX model when $J_{z}=0$ and isotropic XXX model when $J_{z}=J$. Parameters $J, J_{z}$ and $D_{z}$ are dimensionless.

In the standard basis $\{|00\rangle,|01\rangle,|10\rangle,|11\rangle\}$, the Hamiltonian (1) can be expressed as

$$
H=\left(\begin{array}{cccc}
J_{z} & 0 & 0 & 0 \\
0 & -J_{z} & 2 J+2 i D_{z} & 0 \\
0 & 2 J-2 i D_{z} & -J_{z} & 0 \\
0 & 0 & 0 & J_{z}
\end{array}\right)
$$

By a straightforward calculation we can obtain $H$ eigenstates:

$$
\left|\Phi_{1}\right\rangle=|00\rangle
$$




$$
\begin{gathered}
\left|\Phi_{2}\right\rangle=|11\rangle, \\
\left|\Phi_{3}\right\rangle=\frac{1}{\sqrt{2}}\left(e^{i \theta}|01\rangle+|10\rangle\right), \\
\left|\Phi_{4}\right\rangle=\frac{1}{\sqrt{2}}\left(e^{i \theta}|01\rangle-|10\rangle\right),
\end{gathered}
$$

with corresponding eigenvalues:

$$
\begin{gathered}
E_{1}=J_{z}, \\
E_{2}=J_{z}, \\
E_{3}=-J_{z}+2 w, \\
E_{4}=-J_{z}-2 w,
\end{gathered}
$$

where $w=\sqrt{J^{2}+D_{z}^{2}}, \theta=\arctan \left(\frac{D_{z}}{J}\right)$.

The state of a spin chain system at thermal equilibrium is $\rho(T)=\frac{\exp (-\beta H)}{Z}$, where $Z=\operatorname{tr}[\exp (-\beta H)]$ is the partition function of the system, $H$ is the system Hamiltonian and $\beta=\frac{1}{K_{B} T}$, with $T$ is temperature and $K_{B}$ is the Boltzmann costant which we take equal to 1 for simplicity. Here $\rho(T)$ represents a thermal state, so the entanglement in the thermal state is called thermal entanglement [28]. In the above standard basis, the state of this system at thermal equilibrium can be expressed as:

$$
\rho(T)=\frac{1}{Z}\left(\begin{array}{cccc}
e^{-\beta J_{z}} & 0 & 0 & 0 \\
0 & u & v e^{i \theta} & 0 \\
0 & v e^{-i \theta} & u & 0 \\
0 & 0 & 0 & e^{-\beta J_{z}}
\end{array}\right),
$$

where $u=\frac{1}{2}\left(1+e^{4 \beta w}\right) e^{\beta\left(J_{z}-2 w\right)}, \quad v=\frac{1}{2}(1-$ $\left.e^{4 \beta w}\right) e^{\beta\left(J_{z}-2 w\right)}$, and $Z=2 e^{-\beta J_{z}}\left[1+e^{2 \beta J_{z}} \cosh (2 \beta w)\right]$.

In what follows, we consider the concurrence to quantify the amount of entanglement of the above two-qubit system state $\rho(T)$. The concurrence [29, 30] is defined as $C(\rho(T))=$ $\max \left[2 \max \left(\lambda_{i}\right)-\Sigma_{i} \lambda_{i}, 0\right]$, where $\lambda_{i}(i=1,2,3,4)$ are the square roots of the eigenvalues of the matrix $R=\rho S \rho^{*} S$, in which $S=\sigma_{1}^{y} \otimes \sigma_{2}^{y}, \rho$ is the density matrix of the Eq. (5), and the asterisk denote the complex conjugate. After some straightforward calculation, we get

$$
\begin{gathered}
\lambda_{1}=\frac{1}{Z} e^{-\beta J_{z}}, \\
\lambda_{2}=\frac{1}{Z} e^{-\beta J_{z}}, \\
\lambda_{3}=\frac{1}{Z} e^{\beta\left(J_{z}-2 w\right)}, \\
\lambda_{4}=\frac{1}{Z} e^{\beta\left(J_{z}+2 w\right)},
\end{gathered}
$$

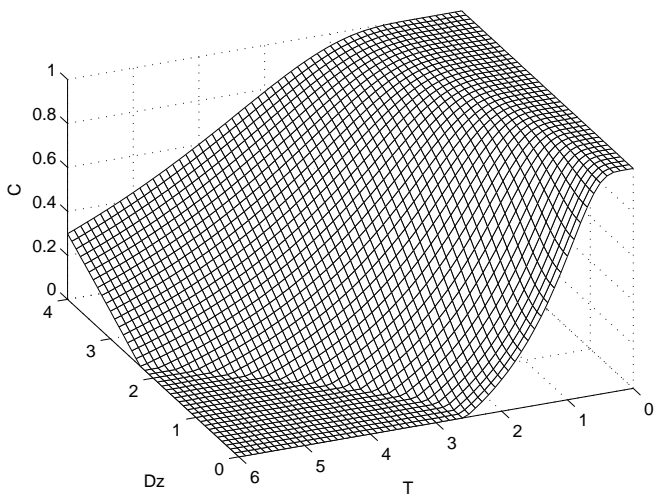

FIG. 1: The concurrence is plotted versus $T$ and $D_{z}$ where the coupling constants $J=1$ and $J_{z}=0.2$.

thus the corresponding concurrence can be expressed as:

$C(\rho(T))=\max \left\{\frac{e^{\beta J_{z}}}{Z}\left(\left|e^{2 \beta w}-e^{-2 \beta J_{z}}\right|-e^{-2 \beta w}-e^{-2 \beta J_{z}}\right), 0\right\}$.

The concurrence is invariant under the substitutions $J \rightarrow-J$ and $D_{z} \rightarrow-D_{z}$, so we can restrict $J>0$ and $D_{z}>0$ without loss of generality. The concurrence ranges from 0 to $1, C(\rho(T))=0$ and $C(\rho(T))=1$ indicate the vanishing entanglement and the maximal entanglement respectively. We can see from Eq. (7) that the entanglement $C(\rho(T))=$ $\frac{e^{\beta J_{z}}}{Z}\left(e^{2 \beta w}-e^{-2 \beta J_{z}}-e^{-2 \beta w}-e^{-2 \beta J_{z}}\right)$ if $J_{z}>-w$, and $C(\rho(T))=0$ if $J_{z}<-w$. Here we analyze the $J_{z}>-w$ case.

When $T=0$, the system is in its ground state. It is easy to find that the ground-state energy is equal to

$$
\begin{gathered}
E_{4}=-J_{z}-2 w, \text { if } J_{z}>-w, \\
E_{1}=E_{2}=J_{z}, \text { if } J_{z}<-w .
\end{gathered}
$$

Thus, the ground state is the disentangled state $\left|\Phi_{1}\right\rangle$ or $\left|\Phi_{2}\right\rangle$ when $J_{z}<-w$, and the ground state is the entangled state $\left|\Phi_{4}\right\rangle$ when $J_{z}>-w$. The entanglement of the ground state $\left|\Phi_{4}\right\rangle$ is the maximal entanglement with $C\left(\left|\Phi_{4}\right\rangle\right)=1$.

As the temperature increases, thermal fluctuation will be introduced into the system, thus the entanglement will be changed due to the mix of the ground states and the excited states. When the temperature is higher than a critical temperature the entanglement is zero. Quantum phase transition happens at the critical temperature $T_{c}$. From Eq. (7), we obtain the following critical temperature equation

$$
e^{\frac{2 J_{z}}{T_{c}}} \sinh \left(\frac{2 w}{T_{c}}\right)=1
$$

To see the change of the entanglement in detail, we analyze the concurrence of Eq. (7). By fixing some parameters we can know the roles of the other parameters and the variation of the entanglement. In Fig. 1, the thermal entanglement is 


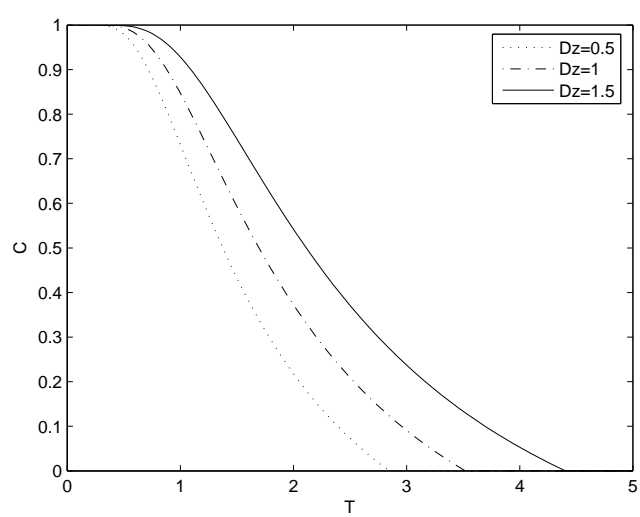

FIG. 2: The concurrence is plotted as a function of the temperature $T$ for different $D_{z}$, here $J=1$ and $J_{z}=0.2$.

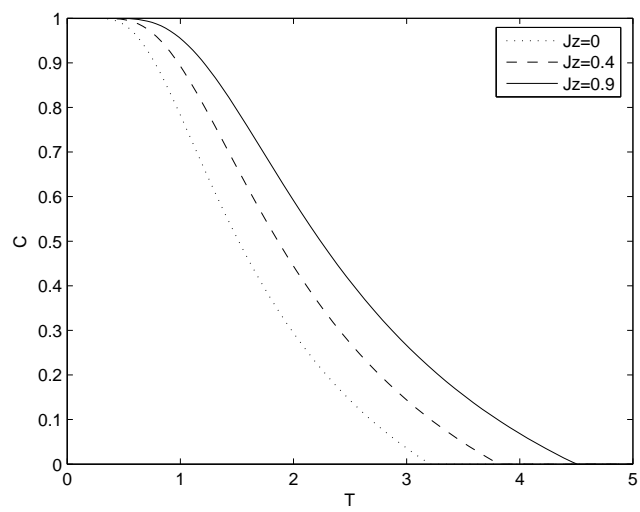

FIG. 3: The concurrence versus $T$ for different $J_{z}$ in the system with z-component parameter $D_{z}$, here $J=1$ and $D_{z}=1$.

plotted versus $T$ and $D_{z}$ where the coupling constants $J=1$ and $J_{z}=0.2$. From the figure, it's obvious that the increased temperature $T$ can decrease the entanglement. The reason is the mixing of the maximally entangled state with other states. In addition, it is easy to see that the entanglement will increase as the DM interaction parameter $D_{z}$ increases. Fig. 2 demonstrates the concurrence versus temperature for different DM coupling parameter $D_{z}$ when $J=1$ and $J_{z}=0.2$. It shows that the concurrence will decrease with increasing temperature $T$ and increase with increasing $D_{z}$ for a certain temperature. The critical temperature $T_{c}$ determined by Eq. (9) is dependent on $D_{z}$, increasing $D_{z}$ can increase the critical temperature above which the entanglement vanishes. Similarly, Fig. 3 shows the concurrence versus temperature for different anisotropic coupling parameter $J_{z}$ when $J=1$ and $D_{z}=1$. We can see that the entanglement decrease with the increase of temperature, and by increasing $J_{z}$, the critical temperature is increased and the entanglement is enhanced for a certain temperature.

So the DM interaction parameter $D_{z}$ and anisotropic coupling coefficient $J_{z}$ are both efficient control parameters of entanglement, by increasing them, we can enhance the entan- glement or increase the critical temperature to slow down the decreace of the entanglement.

\section{XXZ HEISENBERG MODEL WITH DM INTERACTION PARAMETER $D_{x}$}

Here we consider the case of the two-qubit anisotropic Heisenberg XXZ chain with DM interaction parameter $D_{x}$. The Hamiltonian is

$$
H^{\prime}=J \sigma_{1}^{x} \sigma_{2}^{x}+J \sigma_{1}^{y} \sigma_{2}^{y}+J_{z} \sigma_{1}^{z} \sigma_{2}^{z}+D_{x}\left(\sigma_{1}^{y} \sigma_{2}^{z}-\sigma_{1}^{z} \sigma_{2}^{y}\right),
$$

where $D_{x}$ is the x-component parameter of DM interaction, $J, J_{z}$ and $\sigma^{i}(i=x, y, z)$ are the same as those in Section II. Parameters $D_{x}, J$ and $J_{z}$ are dimensionless.

In the standard basis $\{|00\rangle,|01\rangle,|10\rangle,|11\rangle\}$, the Hamiltonian (10) can be rewritten as

$$
H^{\prime}=\left(\begin{array}{cccc}
J_{z} & i D_{x} & -i D_{x} & 0 \\
-i D_{x} & -J_{z} & 2 J & i D_{x} \\
i D_{x} & 2 J & -J_{z} & -i D_{x} \\
0 & -i D_{x} & i D_{x} & J_{z}
\end{array}\right) .
$$

After a straightforward calculation we obtain $H^{\prime}$ eigenstates:

$$
\begin{aligned}
& \left|\Psi_{1}\right\rangle=\frac{1}{\sqrt{2}}(|00\rangle+|11\rangle), \\
& \left|\Psi_{2}\right\rangle=\frac{1}{\sqrt{2}}(|01\rangle+|10\rangle),
\end{aligned}
$$

$\left|\Psi_{3}\right\rangle=\frac{1}{\sqrt{2}}(-i \sin \phi|00\rangle+\cos \phi|01\rangle-\cos \phi|10\rangle+i \sin \phi|11\rangle)$

$\left|\Psi_{4}\right\rangle=\frac{1}{\sqrt{2}}(-i \sin \varphi|00\rangle+\cos \varphi|01\rangle-\cos \varphi|10\rangle+i \sin \varphi|11\rangle)$,

with corresponding eigenvalues:

$$
\begin{gathered}
E_{1}^{\prime}=J_{z}, \\
E_{2}^{\prime}=2 J-J_{z}, \\
E_{3}^{\prime}=-J+w^{\prime}, \\
E_{4}^{\prime}=-J-w^{\prime},
\end{gathered}
$$

where $\phi=\arctan \left(\frac{2 D_{x}}{J+J_{z}-w^{\prime}}\right), \varphi=\arctan \left(\frac{2 D_{x}}{J+J_{z}+w^{\prime}}\right)$, and $w^{\prime}=\sqrt{\left(J+J_{z}\right)^{2}+4 D_{x}^{2}}$.

For convenience of analysis, we assume $J_{z} \leqslant J$ in this section (for the case $J_{z}>J$ we can get some similar results). Here, it is easy to see that the system ground-state energy is $E_{4}^{\prime}=-J-w^{\prime}$, thus the corresponding ground state $\left|\Psi_{4}\right\rangle$ is the maximally entangled state with $C\left(\left|\Psi_{4}\right\rangle\right)=1$. In fact the 
four eigenstates in Eq. (12) are all the maximally entangled states, this phenomenon indicates that the ground states have more entanglement in this system than the system of Eq. (1).

At thermal equilibrium the density matrix of this two-qubit spin chain system has the following form:

$$
\rho^{\prime}(T)=\frac{\exp \left(-\beta H^{\prime}\right)}{Z^{\prime}}=\frac{1}{2 Z^{\prime}}\left(\begin{array}{cccc}
\mu_{+} & -\xi & \xi & \mu_{-} \\
\xi & \nu_{+} & \nu_{-} & -\xi \\
-\xi & \nu_{-} & \nu_{+} & \xi \\
\mu_{-} & \xi & -\xi & \mu_{+}
\end{array}\right)
$$

where $Z^{\prime}=2 e^{-\beta J} \cosh \left[\beta\left(J-J_{z}\right)\right]+2 e^{\beta J} \cosh \left(\beta w^{\prime}\right)$ is the partition function of the system, $H^{\prime}$ is the system Hamiltonian and $\beta=\frac{1}{K_{B} T}$ with the Boltzmann costant $K_{B} \equiv$ 1. $\mu_{ \pm}=e^{-\beta J_{z}} \pm\left(e^{\beta\left(J-w^{\prime}\right)} \sin ^{2} \phi+e^{\beta\left(J+w^{\prime}\right)} \sin ^{2} \varphi\right)$, $\nu_{ \pm}=e^{\beta\left(J_{z}-2 J\right)} \pm\left(e^{\beta\left(J-w^{\prime}\right)} \cos ^{2} \phi+e^{\beta\left(J+w^{\prime}\right)} \cos ^{2} \varphi\right)$, and $\xi=i e^{\beta\left(J-w^{\prime}\right)} \sin \phi \cos \phi+i e^{\beta\left(J+w^{\prime}\right)} \sin \varphi \cos \varphi$.

In what follows, we calculate the square roots of the eigenvalues of the matrix $R^{\prime}=\rho^{\prime} S \rho^{\prime} * S$, where $\rho^{\prime *}$ is the complex conjugate of $\rho^{\prime}$ and $S=\sigma_{1}^{y} \otimes \sigma_{2}^{y}$. The square roots of the eigenvalues of the matrix $R^{\prime}$ are:

$$
\begin{gathered}
\lambda_{1}^{\prime}=\frac{1}{Z^{\prime}} e^{\beta\left(J_{z}-2 J\right)}, \\
\lambda_{2}^{\prime}=\frac{1}{Z^{\prime}} e^{-\beta J_{z}}, \\
\lambda_{3}^{\prime}=\frac{1}{Z^{\prime}} e^{\beta J}\left[\cosh \left(\beta w^{\prime}\right)+\sqrt{\cosh ^{2}\left(\beta w^{\prime}\right)-1}\right] \\
\lambda_{4}^{\prime}=\frac{1}{Z^{\prime}} e^{\beta J}\left[\cosh \left(\beta w^{\prime}\right)-\sqrt{\cosh ^{2}\left(\beta w^{\prime}\right)-1}\right] .
\end{gathered}
$$

According to the methods in [29, 30], when $J_{z} \leqslant J$ we obtain the corresponding concurrence

$$
\begin{aligned}
C\left(\rho^{\prime}(T)\right) & =\max \left\{\frac { 1 } { Z ^ { \prime } } \left[\mid e^{\beta J}\left(\cosh \left(\beta w^{\prime}\right)+\sqrt{\cosh ^{2}\left(\beta w^{\prime}\right)-1}\right)\right.\right. \\
& -e^{-\beta J_{z}} \mid-e^{\beta J}\left(\cosh \left(\beta w^{\prime}\right)-\sqrt{\cosh ^{2}\left(\beta w^{\prime}\right)-1}\right) \\
& \left.\left.-e^{\beta\left(J_{z}-2 J\right)}\right], 0\right\} .
\end{aligned}
$$

To analyze the role of parameters and the variation of the entanglement, we restrict $D_{x}>0, J_{z}>0$ and $J>0$. In Fig. 4, the concurrence is plotted versus $T$ and $D_{x}$ when the coupling constants $J=1$ and $J_{z}=0.2$. It is evident that increasing temperature will decrease the entanglement, and increasing $D_{x}$ will enhance the entanglement and increase the critical temperature $T_{c}^{\prime}$ which is determined by Eq. (16). Fig. 5 demonstrates that the concurrence versus $T$ for different $J_{z}$ with x-component parameter $D_{x}=1$ and $J=1$, it is easy to find that increasing $J_{z}$ can increase the critical temperature and enhance the entanglement for a certain temperature. So $D_{x}$ and $J_{z}$ are both efficient control parameters of entanglement, too.

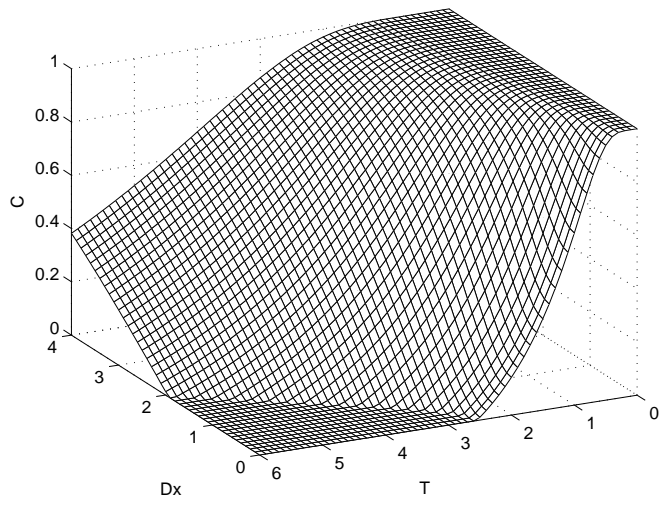

FIG. 4: The concurrence is plotted versus $T$ and $D_{x}$ where the coupling constants $J=1$ and $J_{z}=0.2$.

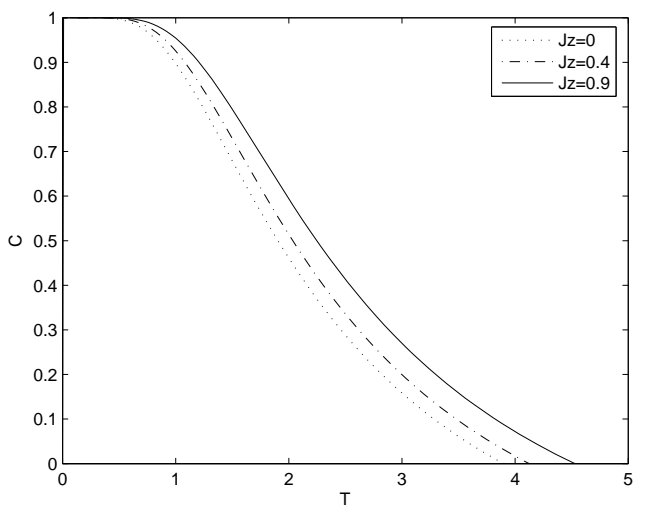

FIG. 5: The concurrence versus $T$ for different $J_{z}$ in the system with x-component parameter $D_{x}$, here $J=1$ and $D_{x}=1$.

\section{THE COMPARISON BETWEEN THE TWO DM INTERACTION COMPONENT PARAMETERS}

From Section II and Section III, we know that the $\mathrm{x}$ component parameter $D_{x}$ and the z-component parameter $D_{z}$ of DM interaction have the similar qualities. They are both efficient control parameters of entanglement, increasing them can enhance the entanglement or increase the critical temperature to slow down the decrease of entanglement.

In this section, we mainly analyze the differences between $\mathrm{X}$-component parameter and $\mathrm{z}$-component parameter. We note that there is less disentanglement region in Fig. 4 than Fig. 1, where $J=1$ and $J_{z}=0.2$, and the temperature and the spinorbit coupling parameter have the same range. We also see that increasing $\mathrm{x}$-component parameter $D_{x}$ can make the entanglement increase more rapidly, for example, when $T=6$ the concurrence increases more rapidly in Fig. 4 than Fig. 1. These phenomena denote that X-component parameter $D_{x}$ has a more remarkable influence than the z-component parameter $D_{z}$. In Fig. 6, the concurrence is plotted as a function of the temperature $T$ for $D_{z}=2$ and $D_{x}=2$ with $J=1$ 


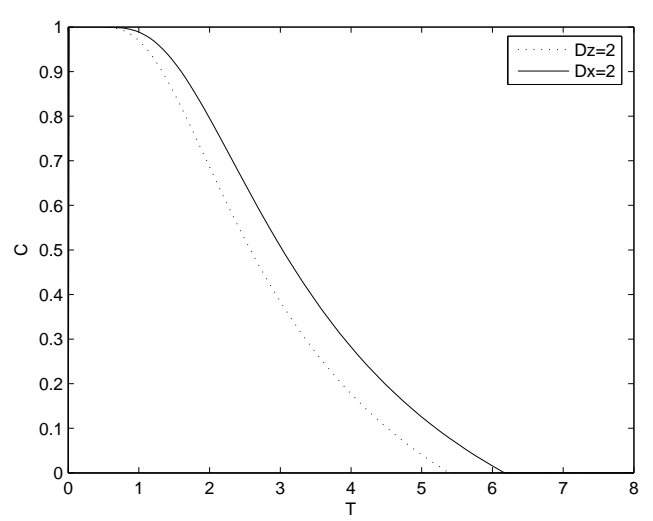

FIG. 6: The concurrence is plotted as a function of the temperature $T$ for $D_{z}=2$ and $D_{x}=2$, here $J=1$ and $J_{z}=0.2$.

and $J_{z}=0.2$. It is easy to see that for the same $D_{x}$ and $D_{z}$, x-component parameter $D_{x}$ has a higher critical temperature and more entanglement for a certain temperature than zcomponent parameter $D_{z}$. We show distinctly the differences between different DM interaction parameters in Fig. 6. So using the DM interaction parameters in different directions, we can increase the entanglement and the critical temperature with different efficiencies.

\section{DISCUSSION}

The entanglement of a two-qubit Heisenberg XXZ system with different DM interaction parameters have been investi- gated. The DM interaction parameter and coupling coefficient $J_{z}$ are efficient control parameters of the entanglement. By increasing the parameters, we can enhance the entanglement or increase the critical temperature to slow down the decreace of the entanglement. In addition, we have also investigated the differences between $\mathrm{x}$-component DM interaction parameter $D_{x}$ and z-component parameter $D_{z}$. Entanglement can be increased more rapidly by increasing $D_{x}$ than $D_{z}$. When $D_{x}$ and $D_{z}$ have the same value, $D_{x}$ has a higher critical temperature than $D_{z}$. Thus, by changing the direction of DM interaction, we can obtain a more efficient control parameter to increase the entanglement and the critical temperature.

\section{Acknowledgments}

This work is supported by National Natural Science Foundation of China (NSFC) under Grant Nos: 60678022 and 10704001, the Specialized Research Fund for the Doctoral Program of Higher Education under Grant No. 20060357008, Anhui Provincial Natural Science Foundation under Grant No. 070412060, the Talent Foundation of Anhui University, and Anhui Key Laboratory of Information Materials and Devices (Anhui University).

[1] C. H. Bennett and S. J. Wiesner, Phys. Rev. Lett. 69 (1992) 2881; M. Murao, D. Jonathan, M. B. Plenio and V. Vedral, Phys. Rev. A 59 (1999) 156.

[2] M. A. Neilsen and I. L. Chuang, Quantum computation and quantum information (Cambridge University Press), Cambridge, UK, 2000.

[3] X. Wang, Phys. Rev. A 64 (2001) 012313.

[4] K. M. OConnor and W. K. Wootters, Phys. Rev. A 63 (2001) 052302 .

[5] G. L. Kamta and A. F. Starace, Phys. Rev. Lett. 88 (2002) 107901.

[6] D. V. Khveshchenko, Phys. Rev. B 68 (2003) 193307.

[7] T. Senthil, J. B. Marston and Matthew P. Fisher, Phys. Rev. B 60 (1999) 4245.

[8] M. Nishiyama, Y. Inada and Guo-qing Zheng, Phys. Rev. Lett. 98 (2007) 047002.

[9] B. Trauzettel, Denis V. Bulaev, Daniel Loss and Guido Burkard, Nature Phys. 3 (2007) 192.

[10] R. Hanson, L. P. Kouwenhoven, J. R. Petta, S. Tarucha and L. M. K. Vandersypen, Rev. Mod. Phys. 79 (2007) 1217.

[11] F. Bodoky and M. Blaauboer, Phys. Rev. A 76 (2007) 052309.

[12] D. Porras and J. I. Cirac, Phys. Rev. Lett. 92 (2004) 207901.

[13] X. X. Yi, H. T. Cui and L. C. Wang, Phys. Rev. A 74 (2006) 054102 .
[14] Y. Sun, Y. Chen and H. Chen, Phys. Rev. A 68 (2003) 044301.

[15] G. F. Zhang and S. S. Li, Phys. Rev. A 72 (2005) 034302.

[16] M. Asoudeh and V. Karimipour, Phys. Rev. A 71 (2005) 022308.

[17] X. Q. Su and A. M. Wang, Phys. Lett. A 369 (2007) 196.

[18] Z. N. Gurkan and O. K. Pashaev, e-print quant-ph/0705.0679 and quant-ph/0804.0710.

[19] Y. Yeo, Phys. Rev. A 66062312 (2002).

[20] X. Hao and S. Q. Zhu, Phys. Lett. A 338 (2005) 175.

[21] L. Campos Venuti, S. M. Giampaolo, F. Illuminati, and P. Zanardi, Phys. Rev. A 76 (2007) 052328.

[22] G. F. Zhang, Phys. Rev. A 75 (2007) 034304.

[23] F. Kheirandish et al., Phys. Rev. A 77 (2008) 042309.

[24] D. Loss and D. P. DiVincenzo, Phys. Rev. A 57 (1998) 120.

[25] D. A. Lidar, D. Bacon, and K. B. Whaley, Phys. Rev. Lett. 82 (1999) 4556.

[26] D. P. Divincenzo, D. Bacon, J. Kempe, G. Burkard, and K. B. Whaley, Nature (London) 408 (2000) 339.

[27] L. F. Santos, Phys. Rev. A 67 (2003) 062306.

[28] M. A. Nielsen, arXiv:quant-ph/0011036

[29] S. Hill and W. K. Wootters, Phys. Rev. Lett. 78 (1997) 5022.

[30] W. K. Wootters, Phys. Rev. Lett. 80 (1998) 2245. 